\title{
Atorvastatin Calcium Anhydrous
}

National Cancer Institute

\section{Source}

National Cancer Institute. Atorvastatin Calcium Anhydrous. NCI Thesaurus. Code C78759.

An anhydrous calcium salt form of atorvastin, a synthetic lipid-lowering agent. 